\title{
Behavioral variability as a function of response topography and reinforcement contingency
}

\author{
LAURA MORGAN and ALLEN NEURINGER \\ Reed College, Portland, Oregon
}

\begin{abstract}
Long-Evans rats were reinforced for generating variable sequences of responses on two operanda. The current sequence of four left and right responses was required to differ from each of the previous five sequences. Variability under this VARY schedule was compared with that under a YOKE control schedule where reinforcement was independent of the sequences. Three different response topographies were compared: two levers were pressed in one case, two keys pushed in another, and two wires pulled in a third. Both reinforcement contingency (VARY vs. YOKE) and response topography (leverpress, key push, and wire pull) significantly influenced sequence variability. As is the case for operant dimensions, behavioral variability is jointly controlled by reinforcement contingency and response topography.
\end{abstract}

When a dimension of behavior both controls reinforcement and is controlled by reinforcement, the behavior is generally referred to as "instrumental" or "operant." For example, when reinforcement is contingent upon a particular class of responses, such as leverpresses, and presses increase in probability, the press response is called an operant. So, too, for response location, topography, latency, rate, probability, and force. Behavioral variability may also be an operant. For example, pigeons learned to generate highly variable interresponse times when reinforcement was contingent upon the least frequent interresponse time (Blough, 1966), rats generated variable sequences of responses across two levers when reinforcement depended upon relatively high frequencies of switches (Bryant \& Church, 1974), dolphins emitted unusual flips and jumps when reinforced for behaviors never before observed by the experimenters (Pryor, Haag \& O'Reilly, 1969), children created unusual drawings and block constructions when reinforced for so doing (Holman, Goetz \& Baer, 1977), and adults learned to generate quasi-random sequences of numbers when given feedback indicating how closely their numbers approximated a computer-based random sequence (Neuringer, 1986). While each of these studies is consistent with the hypothesized operant nature of behavioral variability, none of them demonstrated that reinforcement was necessary for the variability. As pointed out by Schwartz (1982), variability is often elicited or induced by intermittent reinforcement or other aspects of an experimental situation.

Page and Neuringer (1985) performed the necessary comparisons. Experimentally naive pigeons were trained to peck two response keys eight times-constituting one

This work was partly derived from an undergraduate senior thesis submitted by Laura Morgan to Reed College and was partly supported by National Science Foundation Grant BNS-8707992. Reprints may be obtained from Allen Neuringer, Department of Psychology, Reed College, Portland, OR 97202. trial-for grain reward. If the sequence of $L(e f t)$ and $R$ (ight) responses in the current trial differed from each of the sequences in the previous 50 trials, then the current trial was followed by food; for example, reinforcement was provided if the current sequence was LLRLRLLL and that sequence had not occurred during the 50 previous trials. If the current sequence repeated any one of the previous 50 , the trial ended with a brief time-out. Pigeons generated highly variable response sequences under these contingencies. When the same birds were then reinforced independently of sequence variability in a "self-yoke" phase, but with exactly the same frequency and distribution of reinforcers as in the preceding variability phase, behavioral variability decreased significantly. This was strong evidence that variability is an operant dimension: high levels of variability were generated only when reinforcement was contingent upon such variability. Adding further support, sequence variability increased in response to increasing schedule demands, for example, when the contingencies required that the current sequence differ from each of the last 15 sequences, variability was greater than when the contingencies required that the current sequence differ from each of the last 5 sequences (see also Machado, 1989). Furthermore, as with other operant dimensions, variability was controlled by discriminative stimuli: in the presence of red keylights, the pigeons emitted highly variable sequences, whereas in the presence of blue lights, they learned to emit a single sequence.

The present research further compares the variability dimension with other operant dimensions by examining it as a function of response topography. The control exerted by a reinforcer over an operant response is influenced by the topography of the response. For example, food reinforcers exert little control over face washing, body scratching, and scent marking in hamsters, whereas digging, scrabbling, and rearing are precisely controlled (e.g., Shettleworth, 1973, 1975). In other cases, functional relationships across different response topographies 
are often similar to one another, with quantitative, rather than qualitative, differences observed (Hemmes, 1975; Lejeune \& Jasselette, 1986; Morgan, 1987; Richardson, 1979; Richardson \& Clark, 1976).

Our first experiment reinforced variable sequences of leverpresses in one phase, key pushes in another, and wire pulls in a third. Each rat was trained with each of these operanda in counterbalanced order. To assess the degree to which variability depended upon contingent reinforcement, a self-yoked control condition, used for comparison, provided reinforcement independently of sequence variation. If variability serves as an operant dimension, sequence variation should be greater when reinforcement is contingent upon it than when reinforcement is provided independently of variation. Secondly, if response topography is important, sequence variation should differ as a function of the three operanda.

\section{EXPERIMENT 1}

\section{Method}

Subjects. Six male Long-Evans rats, 12 months old at the beginning of the experiment, received free access to food for $2 \mathrm{~h}$ each day following experimental sessions and, except during these sessions, had continuous access to water.

Apparatus. Two modified Gerbrands operant chambers, $31 \times 26$ $\times 38 \mathrm{~cm}$, were housed inside sound- and light-attenuating boxes. A pellet tray, $3 \mathrm{~cm}$ above the floor and centered on the right wall, provided access to a single $45-\mathrm{mg}$ Noyes animal feed pellet as reinforcement. Two levers, $5 \mathrm{~cm}$ long, $6.5 \mathrm{~cm}$ above the floor, and spaced $9 \mathrm{~cm}$ apart, were located on the right wall, equidistant from the pellet tray. Three Gerbrands pigeon keys, spaced $6 \mathrm{~cm}$ apart and $12 \mathrm{~cm}$ above the chamber floor, were located on the left wall; two of them (middle and left) could be transilluminated with white light during the "key" phase of the experiment. Directly beneath the middle key was a dipper port which was not used in the present experiment. During the "wire" phase of the experiment, two overhead wires, with $0.75-\mathrm{cm}$ loops on their bottom ends, extended $20 \mathrm{~cm}$ from the ceiling of the chamber. The wires were spaced $18 \mathrm{~cm}$ apart and centered between right and left chamber walls. Each chamber was connected via Metaresearch interface modules to a Macintosh computer with programs written in True Basic.

The chambers provided access to a single set of operanda during any given session-two levers, two keys, or two wires, respectively - this being accomplished by insertion of false walls, made of particle board, as follows (see Figure 1). When levers were the

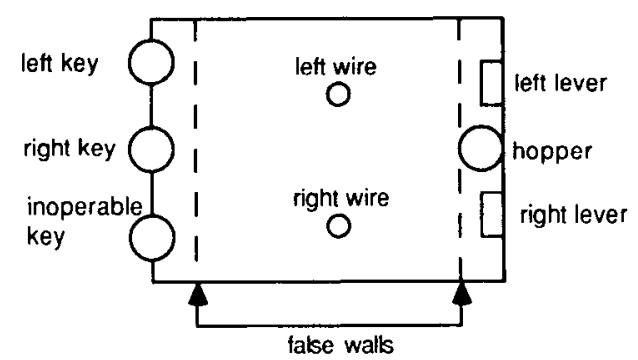

Figure 1. The experimental chamber for Experiment 1. Access to only one pair of operanda - levers, keys, or wires - was provided by inserting false walls. The pellet hopper was accessible during all conditions; when the right wall blocked the two levers, an opening provided access to the hopper. effective operanda, a wall covered the keys and the wires were absent. When keys were the effective operanda, the keys were lighted, a wall covered the two levers, and the wires were absent. When wires were the effective operanda, two wires hung from the ceil ing and walls covered keys and levers. In all conditions, the same pellet tray on the right wall was used. The particle-board false wall permitted access to the pellet tray through a wide opening.

Procedure. The subjects were trained, in counterbalanced order, to press each of two levers, push each of two keys, and pull each of two overhead wires for food reinforcement, and were given preliminary training to respond variably on these operanda (see McElroy \& Neuringer, in press). The two main independent variables, namely response topography and reinforcement contingency, were then systematically manipulated as follows.

The first question asked was whether topography of response influenced behavioral variability. Each subject experienced each of the three operanda in three separate phases. The order of experience was counterbalanced across subjects so that 2 subjects began with levers, 2 with keys, and 2 with wires; then a quasi-random order determined the next operandum, until all subjects had received each of the three separate phases. Under a VARY, lag 5 schedule of reinforcement (see McElroy \& Neuringer, in press; Page \& Neuringer, 1985), a trial consisted of four responses on the two available operanda. If the current sequence of $L$ and $R$ responses differed from each of the sequences in the previous five trials, the trial terminated with reinforcement. If the current sequence repeated any of those in the last five trials, reinforcement was not provided.

At the start of each trial, the chamber was illuminated by the overhead houselight. (When keys were the operanda, the left and middle keys were also transilluminated with white light; see Figure 1.) Following an effective response to either operandum, the chamber was darkened for $0.3 \mathrm{sec}$ and a $2000-\mathrm{Hz}, 0.3-\mathrm{sec}$ tone sounded. During this interval, to be referred to as the interresponse interval (IRI), responses were ineffective and not counted toward completion of the four-response trial. Following the IRI, the chamber was again illuminated and the next effective response could be made. The fourth response terminated the trial with a 1-sec end-of-trial period (EOT), during which the chamber was dark and a pulsating $2000-\mathrm{Hz}$ tone sounded. As with IRI responses, responses during the EOT period had no effect. If the current sequence differed from each of the previous five, a 45-mg Noyes animal food pellet was dispensed at the end of the EOT. If the current trial repeated any of the previous five sequences, no pellet was dispensed. In either case, a new trial followed immediately. Thus, in brief, each trial consisted of four effective responses. A 0.3-sec IRI followed each of the first three responses and a 1-sec end-of-trial interval followed the fourth response. Only when the sequence of Ls and Rs differed from the previous five sequences was a food pellet presented. This VARY schedule enabled us to assess whether response topography influenced sequence variability.

The second question asked was whether contingent reinforcement was necessary for the variability observed. Each of the three operandum phases (lever, key, and wire) was further divided into three conditions: In the first, variability was required for reinforcement (VARY 1) under the contingencies just described; in the second condition, reinforcement was presented independently of behavioral variability (YOKE), as will be described below; and in the third, there was a return to reinforcement of variability (VARY2).

In the YOKE condition, reinforcement was provided independently of the current sequence of responses (see Page \& Neuringer, 1985). As under the VARY contingencies, trials consisted of four responses, the IRI was $0.3 \mathrm{sec}$ and the EOT was $1 \mathrm{sec}$. However, reinforcement depended not on a subject's current response sequence, but on its sequence during the previous VARY condition. For example, reinforcement during the first session of the YOKE condition depended upon the first session of the VARY condition, such that the same number of reinforcements was presented in precisely the same 
series of reinforced and nonreinforced trials as in the first session of VARY. A subject was reinforced in a given trial only if it had received reinforcement during the analogous trial under VARY. For example, if the subject's 25 th trial of VARY training had been reinforced, its 25 th trial during yoKE would also be reinforced, regardless of the current sequence of four responses. This was true for each session of YOKE: Reinforcement depended not on current sequence variability, but on reinforcement in the analogous VARY session. Since there were 15 YOKE sessions and only 9 sessions during the first VARY condition, during the 10th session of YOKE, a subject was yoked again to the first VARY session, that is, the selfyoke contingency looped back to the beginning.

A return to VARY, lag 5 contingencies immediately followed YOKE, this VARY 2 phase being identical to VARY1.

Thus, in brief, each of the 6 subjects experienced the three operanda-lever, key, and wire--in counterbalanced order and, with each operandum phase, three contingencies-VARY, YOKE, and return to VARY. The two VARY conditions were identical. The YOKE condition was also identical to VARY except that variable sequences of responses, while permitted, were not required for reinforcement. The number of sessions were: in Phase 1, VARY1 ( 9 sessions), YOKE (15 sessions), vary 2 (11 sessions); in Phase 2, varyl (10 sessions), YOKE (15 sessions), VARY2 (11 sessions); and in Phase 3, vaRY1 (11 sessions), YOKE (16 sessions), VARY 2 (8 sessions). All sessions ended after the 100th trial.

Data Analyses. Percent variation and $U$ value were the two major dependent measures. Percent variation is the percentage of trials in a session that met the VARY lag 5 contingency (number of trials that differed from each of the previous 5 trials, divided by total trials in a session multiplied by 100 ). Note that during the VARY condition, percent variation was identical to percentage of reinforced trials. In the YOKE condition, percent variation was an index of the percentage of trials that would have been reinforced if a VARY contingency had been in effect. The second measure was $U$ value, which provided a measure of overall sequence variability. Given four $\mathrm{L}$ and $R$ responses per trial, 16 different patterns were possible $\left(2^{4}\right)$. According to the $U$ statistic (Page \& Neuringer, 1985), if all sequences occurred equally often in a given session, $U$ would equal 1.0; if only a single pattern occurred throughout a session, for example, LLLL, then $U$ would equal 0.0 . Thus, as the probability distribution of the 16 sequences flattened, $U$ value approached 1.0 . Averages over the last three sessions at each condition were used throughout. Repeated measures, two-way analyses of variance evaluated the three operanda phases by three contingency conditions.

\section{Results}

The first finding was that response topography influenced behavioral variability. Figure 2 (top), shows percentages of trials that met the lag 5 variability criterion for each of the three response topographies during the VARY, YOKE, and return-to-VARY conditions. Shown in the figure are averages of all subjects and standard errors. To simplify statistical analysis, performances in the two VARY conditions were averaged and the average used in all comparisons. There was a significant main effect of response topography $[F(2,10)=11.373, p=.003]$, with higher overall levels of sequence variability on levers than on keys $[F(1,5)=7.565, p=.040]$ and wires $[F(1,5)$ $=18.441, p=.008]$, but the latter two operanda not differing from one another $[F(1,5)=4.370]$. Thus, topography of response influenced behavioral variability.

The second finding was that contingencies of reinforcement-VARY versus YOKE-also influenced variability. Figure 2 (top) shows that sequence variability was higher
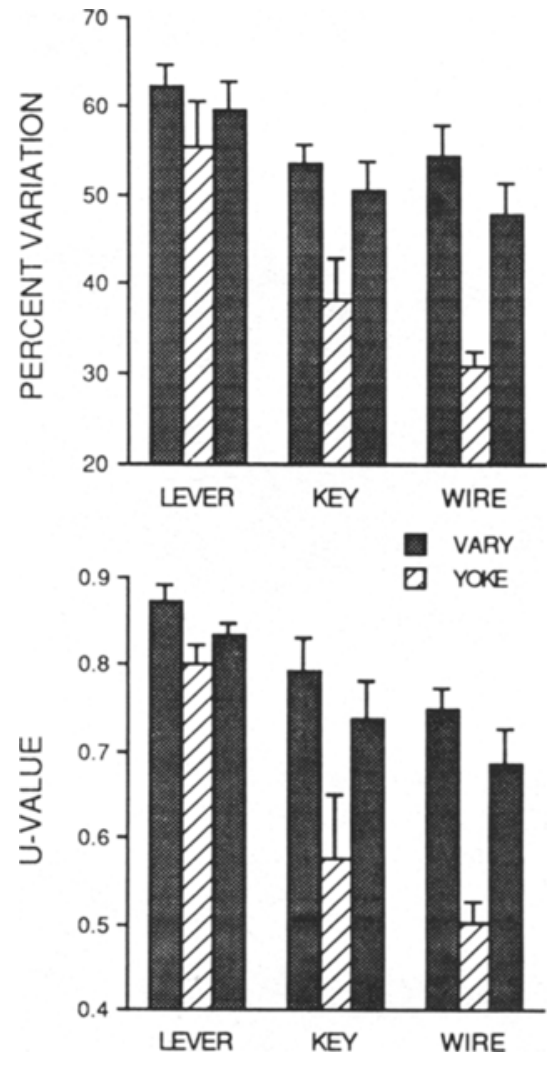

Figure 2. Average percent variations (top) and U-values (bottom) as functions of response topography-lever-press, key-push, and wire-pull-and reinforcement contingency, VARY (grey) and YOKE (striped), in Experiment 1. Standard errors are shown. Data are from the last three sessions of each condition.

under VARY contingencies than under YOKE contingencies $[F(1,5)=166.923, p=.000]$. Although the interaction between response topography and reinforcement contingency was not significant $[F(2,10)=3.142]$, Figure 2 (top) suggests that the greatest difference between VARY and YOKE performances occurred for the wire-pull response and the least occurred for leverpress. This apparent difference in the effects of contingency was analyzed in two ways. First, we calculated each subject's ratio of percent variation during YOKE to its percent variation during VARY. These ratios, indicating the decrease in variability due to the YOKE contingencies, changed significantly as a function of response topography $[F(2,10)=$ $3.986, p=.053$ ], with leverpress and wire-pull ratios significantly differing from one another $[F(1,10)=7.906$, $p=.0184]$ and no other comparisons reaching significance. Secondly, we analyzed percent variation under each of the topographies individually. YOKE percent variation was significantly lower than VARY in the key $[F(1,5)$ $=12.306, p=.017]$ and wire $[F(1,5)=53.201, p=$ $.001]$ phases but not in the lever phase $[F(1,5)=3.205]$. Thus, while YOKE contingencies generated lower levels of variability overall, the magnitude and significance of the effects were influenced by the particular topographies. 
An index of overall response variation is given by $U$ value, shown in the lower graph in Figure 2. Although high percent-variation values could be generated if a subject cycled through only five different sequences, high $U$ values depended upon equal distributions across all of the 16 sequences. $U$-value changes were similar to percent variation: topography exerted a significant effect $[F(2,10)$ $=11.754, p=.002]$, as did contingency $[F(1,5)=$ $37.831, p=.002]$, and all other comparisons were similar to those described for percent variation.

\section{EXPERIMENT 2}

Both topography of response and contingency of reinforcement appeared to affect sequence variability. However, the levers in Experiment 1 were located on the same front wall as the reinforcement tray, the wires were located midway between front and rear walls, and the keys were located on the rear wall. Thus, when the levers were the operanda, the required operant responses were close to the reinforcement tray. When the keys were the operanda, the subjects had to turn around and move to the front of the chamber to receive a pellet. The wires were at an intermediate distance. Experiment 2 thus compared sequence variability using keys and levers when the distance relationships between operanda and reinforcement dispenser were identical.

Furthermore, in Experiment 1, the keys were illuminated when keypress responses were used, whereas levers and wires were dark throughout. Since differences in operandum illumination may have played some role, both keys and levers were now unlighted.

Finally, because of the within-subjects design used in Experiment 1, acquisition of sequence variability could not readily be examined. The present experiment therefore compared two groups, one leverpress and one key push. Given the differences in overall levels of sequence variability observed in Experiment 1 as a function of response topography, and the differential effects of contingencies of reinforcement, the question now asked was whether acquisition of operant variability would also differ as a function of topography.

\section{Method}

Subjects. Twelve experimentally naive male Long-Evans rats, 16 months old at the start of the experiment, received $2 \mathrm{~h}$ of access to approximately $25 \mathrm{~g}$ of laboratory rat chow after each experimental session and free access to water except during experimental sessions.

Apparatus. Two converted Gerbrands operant chambers, similar to those in Experiment 1, were used. One chamber was equipped with two levers, the other with two keys. The spatial relationships between the levers and pellet hopper, in the one case, and keys and pellet hopper, in the other, were identical. Similarly, both keys and levers were dark throughout the experiment. A food hopper, providing access to a 45-mg Noyes pellet, was centered on the left wall $1 \mathrm{~cm}$ above the floor in both chambers. The hopper was $2 \mathrm{~cm}$ high $\times 4 \mathrm{~cm}$ wide $\times 4 \mathrm{~cm}$ deep and was set into the wall. A lever was also on the left wall, $5 \mathrm{~cm}$ above the food hopper, but it was inoperative throughout the present study. The lever chamber contained two Gerbrands levers on the right wall, $4 \mathrm{~cm}$ above the floor and $15 \mathrm{~cm}$ apart. A minimum downward force of $25 \mathrm{~g}$ followed by release defined the leverpress response. The key chamber contained two Gerbrands pigeon keys, $4 \mathrm{~cm}$ above the floor, spaced $15 \mathrm{~cm}$ apart and set in the right wall. A minimum force of $15 \mathrm{~g}$ defined the key-push response. Thus, the two chambers were identical ex cept that one contained two levers and the other two keys

Procedure. All rats were shaped to press left and right levers and push left and right keys for food pellets. The order of training was counterbalanced across subjects. The subjects were then randomly assigned to two groups of 6 each, a lever group and a key group, and, from this point on, a given rat experienced only its assigned operandum. One lever-group animal died of a respiratory disease on the 41 st day of the experiment, and its data were excluded from all analyses. This left 6 key and 5 lever subjects. After the two groups were established, three additional preliminary sessions were given, using the same VARY contingencies and parameters as in Experiment 1, except that a lag 0 VARY contingency was in effect; that is, every sequence of four responses was reinforced. The design of the experiment was similar to that of a single phase of Experiment 1, that is, VARY1-YOKE-VARY2. Contingencies and stimulus events were identical to those in Experiment 1. Twenty sessions of VARYl training were followed by 20 sessions of YOKE, and a final 10 sessions of VARY 2 . Thus, the present design involved a comparison across separate lever and key groups and further within-groups comparisons of contingency (VARY1-YOKE-VARY2).

\section{Results}

The first question asked whether lever and key topographies generated different overall levels of variability when distance relationships were controlled. Figure 3 (top) shows that variability was higher in the lever group than in the key group $[F(1,9)=6.756, p=.029]$, thereby replicating the results of Experiment 1.

The second question asked whether VARY contingencies engendered higher levels of sequence variability than did YOKE contingencies. Here, too, the results replicated Experiment 1: reinforcement contingencies exerted a significant effect $[F(1,9)=12.363, p=.007]$, and the interaction between topography and contingency again did not reach significance $[F(1,9)=1.272]$. However, again as in Experiment 1, although the ratios of YOKE to VARY did not differ significantly across these two topographies $[t(9)=1.269]$, when the two topographies were analyzed separately, there was a significant decrement due to the yoking procedure for the key group $[F(1,9)=11.861$, $p=.007]$ but not for the lever group $[F(1,9)=2.614]$. $U$ values, shown in the bottom of Figure 3 , were once again similar to percent variation in terms of all statistical comparisons.

The third question asked whether lever and key topographies generated different learning curves. Figure 4 shows group average percent variations during each of the initial 10 sessions of VARY 1 training. The key group's percent variation was relatively low at first and increased across sessions. On the other hand, variability in the lever group was high to begin with and remained high throughout. Significant main effects of response topography $[F(1,10)=7.61, p=.020]$, sessions $[F(9,90)=5.82$, $p=.000]$, and interaction $[F(9,90)=4.38, p=.000]$ were obtained. The key group learned to generate variable patterns, whereas the lever group entered the experiment responding in a highly variable manner. 

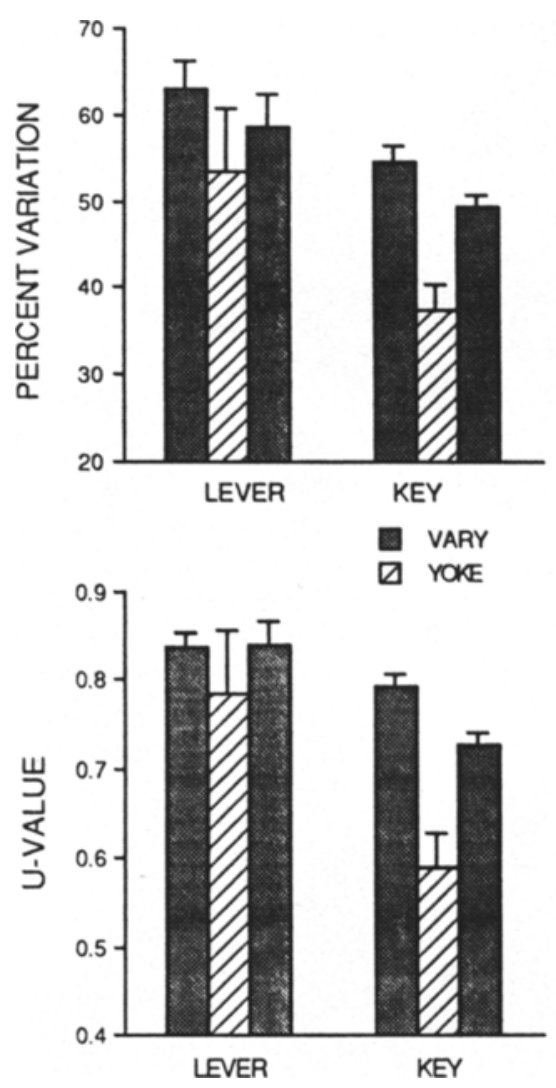

Figure 3. Average percent variations (top) and U-values (bottom) as functions of response topography-lever-press versus key-pushand reinforcement contingency, VARY (grey) and YOKE (striped), in Experiment 2. Standard errors are shown. Data are from the last three sessions of each condition.

Figure 5 shows $U$ values during each session of the experiment for one subject in the key group (upper) and one in the lever group (lower). The key subject's initial $U$ value was lower than 0.4 and it increased to about 0.85 , whereas the lever subject's initial value was relatively high. The figure also shows the decrease in $U$ values during the YOKE condition and reacquisition during return to VARY. These performances are generally representative of group averages (although the lever subject's decrease during YOKE was greater than the average): average key $U$ values increased from 0.577 across the first three VARY 1 sessions to 0.791 across the last three VARY 1 sessions, and then decreased to 0.589 across the last three YOKE sessions. Group average lever values increased from 0.774 to 0.835 during the VARY 1 sessions and decreased to 0.787 during the last three YOKE sessions. Thus average terminal YOKE performances approached average performances at the beginning of the experiment.

\section{DISCUSSION}

Response sequence variability was influenced by the type of operandum used, with leverpresses generating the highest levels of variability, key pushes intermediate levels, and wire pulls the lowest. These results are consistent with findings that show that, given identical reinforcement contingencies, different response topographies occur with differing frequencies (e.g., Anderson \& Shettleworth, 1977; Shettleworth \& Juergensen, 1980; Staddon \& Simmelhag, 1971). Thus, the variability dimension of behavior is influenced by response topography in ways similar to its influence on other dimensions, such as response frequency and probability.

For all topographies, sequence variability was higher when reinforcement was contingent upon the variability (VARY condition) than when reinforcers were provided independently of it (YOKE condition). Since frequencies and distributions of reinforcement were identical under the VARY and YOKE conditions, variability depended upon reinforcement contingent upon it. This was true for both pigeons in Page and Neuringer (1985) and rats in the present experiments. Once again, therefore, behavioral variability is shown to be an operant dimension similar to such other operant dimensions as response rate, probability, force, class and location.

Although there was no significant statistical interaction between topography and contingency in either Experiment 1 or Experiment 2, individual analyses showed that reinforcement contingencies exerted less control over leverpress variability than they did over key-push or wirepull. Response topography influenced the control exerted by reinforcement over sequence variability, a result again analogous to results obtained from other operant dimensions. For example, reinforcement may influence the probability of some classes of responses but not of others (Shettleworth, 1973, 1975). In other instances, response topography is found to have a quantitative, rather than a qualitative, effect (e.g., Hemmes, 1975; Lejeune \& Jasselette, 1986; Morgan, 1987; Richardson, 1979; Richardson \& Clark, 1976).

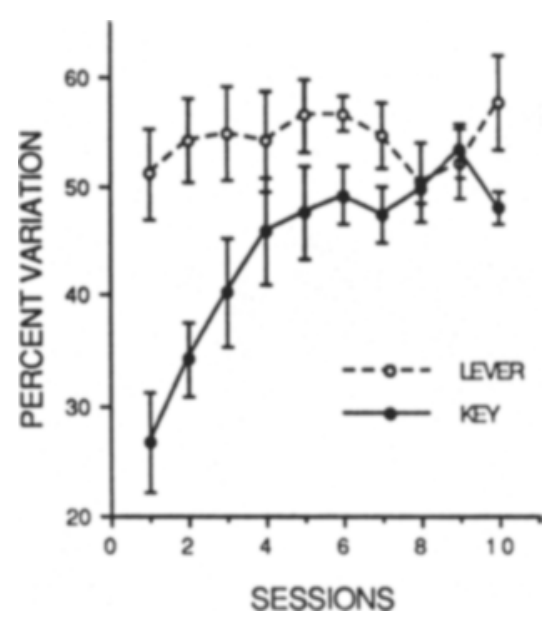

Figure 4. Average percent variations for the lever and key groups during the initial ten sessions of the VARY condition in Experiment 2. Standard errors are shown. 

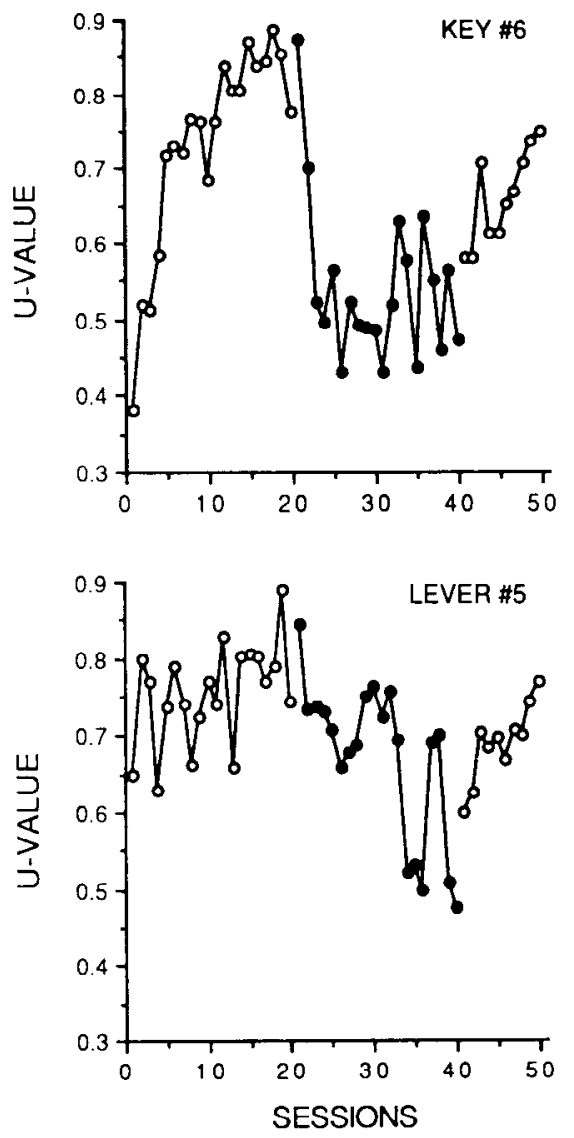

Figure 5. U-values during each session of Experiment 2 for one animal in the key group (upper) and one animal in the lever group (lower). Open circles show performances during the VARY condition; closed circles show performance during the YOKE condition.

All three topographies generated appreciable variability when such variability was not required under the YOKE contingencies. This finding is analogous to demonstrations that other operant dimensions are elicited by aspects of the reinforcement schedule or environment and do not depend entirely on contingent reinforcement. For example, noncontingent reinforcement causes pigeons to generate highly predictable sequences of behaviors (Staddon and Simmelhag, 1971). In neither Staddon and Simmelhag nor the present yoked condition was the resulting behavior necessary for reinforcement. Another example is that of autoshaping, in which pigeons come to peck illuminated keys even though reinforcement does not depend upon such pecks (Brown \& Jenkins, 1968; Downing \& Neuringer, 1976). Results such as these have been characterized as indicating that behaviors are elicited via classical conditioning mechanisms, that the subject is "biologically prepared" to engage in a particular behavior, that there is a high "operant level," or that "superstitious" behaviors are probable. Whatever the explanation, variability appears to be influenced by contextual or eliciting events, as are other operant dimensions. Temperature and size of chamber, internal states induced by deprivation and drugs, and environmental "noise" may also be expected to elicit different levels of behavioral variability.

The high variability elicited by the levers in the YOKE condition was not predicted and may have contributed to a "ceiling effect," making further increments due to VARY reinforcement contingencies difficult to observe. High baseline variability might make the leverpress response particularly sensitive to manipulations by operant contingencies, for variability provides the "operant level" upon which reinforcement contingencies may then work. For example, if the contingencies demanded a particular response sequence, latency, or force, high levels of baseline variability might enable the contingencies quickly to contact and select the behaviors required. On the other hand, when behaviors are sensitive to operant contingencies, we would expect that absence of a requirement for variability would result in a significant decrement in variability, such as that found, for example, for the keypeck response in pigeons (Page \& Neuringer, 1985). Future research must examine whether leverpress responses are generally insensitive to variability contingencies and, if so, why.

However, when reinforcement contingencies require a particular sequence of leverpresses, low variability is observed. For example, McElroy and Neuringer (in press) and Cohen, Neuringer, and Rhodes (in press) reinforced rats for repeating a LLRR sequence of responses and thereby generated relatively low levels of sequence variability. Thus, although leverpress variations occur when not required by the reinforcement contingencies, variability is lowered appreciably when the reinforcement contingencies demand response stereotypy.

In summary, the present research shows that different response topographies elicit different levels of sequence variability and that reinforcement contingencies also control variability. These results are consistent with the hypothesis that sequence variability is an operant dimension, similar to other operants. Future research must explore how topography influences the variability of such other dimensions as response rate, force, location, or class.

\section{REFERENCES}

Anderson, M. C., \& Shettleworth, S. J. (1977). Behavioral adaptation to fixed-interval and fixed-time food delivery in golden hamsters. Joumal of the Experimental Analysis of Behavior, 27, 33-49.

BLough, D. S. (1966). The reinforcement of least frequent interresponse times. Journal of the Experimental Analysis of Behavior, 9, 581-591.

Brown, P. L., \& JeNKINS, H. M. (1968). Autoshaping the pigeon's keypeck. Journal of the Experimental Analysis of Behavior, 11, 1-8.

BRyANT, D., \& ChURCH, R. M. (1974). The determinants of random choice. Animal Learning \& Behavior, 2, 245-248

Cohen, L., Neuringer, A., \& Rhodes, D. (in press). Effects of ethanol on reinforced variations and repetitions by rats under a multiple schedule. Joumal of the Experimental Analysis of Behavior.

Downing, K., \& NeUringer, A. (1976). Autoshaping as a function of prior food presentations. Journal of the Experimental Analysis of Behavior, 26, 463-469.

Hemmes, N. S. (1975). Pigeons' performance under differential reinforcement of low rate schedules depends upon the operant. Learning \& Motivation, 6, 344-357

Holman, J., Goetz, E. M., \& Baer, D. M. (1977). The training of 
creativity as an operant and an examination of its generalization characteristics. In B. Etzel, J. Le Bland, \& D. M. Baer (Eds.), New developments in behavioral research: Theory, method and application (pp. 441-471). Hillsdale, NJ: Erlbaum.

LEJEUNE, H., JASSELETTE, P. (1986). Accurate DRL performance in the pigeon: Comparison between perching and treadle pressing. Animal Learning \& Behavior, 14, 205-211.

MaCHADO, A. (1989). Operant conditioning of behavioral variability using a percentile reinforcement schedule. Journal of the Experimental Analysis of Behavior, 52, 155-166.

McElroy, E., \& Neuringer, A. (in press). Effects of alcohol on reinforced repetitions and reinforced variations in rats. Psychopharmacology.

MoRGAN, B. J. (1987). Response topography and schedule of reinforcement as determinants of dimensional characteristics of rats' operant responses. Unpublished doctoral dissertation, University of Mississippi.

Neuringer, A. (1986). Can people behave "randomly?": The role of feedback. Journal of Experimental Psychology: General, 115, 62-75.

PAGE, S., \& NeURINGER, A. (1985). Variability is an operant. Journal of Experimental Psychology: Animal Behavior Processes, 11, 429-452.

Pryor, K. W., HaAg, R., \& O'ReIlly, J. (1969). The creative porpoise: Training for novel behavior. Journal of the Experimental Analysis of Behavior, 12, 653-661.

Richardson, W. K. (1979). A comparison of the keypeck and treadlepress operants in the pigeon: Differential-reinforcement-of-low-rate yoked variable-interval schedule. Bulletin of the Psychonomic Society, 13, 233-236.
Richardson, W. K., \& ClaRk, D. B. (1976). A comparison of the keypeck and treadle-press operants in the pigeon: Differentialreinforcement-of-low-rate schedule of reinforcement. Journat of the Experimental Analysis of Behavior, 26, 237-256

SCHWARTZ, B. (1982). Reinforcement-induced stereotypy: How not to teach people to discover rules. Journal of Experimental Psychology: General, 111, 23-59.

SHETTLEWORTH, S. J. (1973). Food reinforcement and the organization of behavior in golden hamsters. In R. A. Hinde \& J. StevensonHinde (Eds.), Constraints on learning: Limitations and predispositions. London: Academic Press.

ShetTlewORTH, S. J. (1975). Reinforcement and the organization of behavior in golden hamsters: Hunger, environment, and food reinforcement. Journal of Experimental Psychology: Animal Behavior Processes, 1, 56-87.

Shettleworth, S. J., \& JuergenSEn, M. R. (1980). Reinforcement and the organization of behavior in golden hamsters: Brain stimulation reinforcement for seven action patterns. Journal of Experimen tal Psychology: Animal Behavior Processes, 6, 352-375.

Staddon, J. E. R., \& Simmelhag, V. L. (1971). The "superstition" experiment: A reexamination of its implications for the principles of adaptive behavior. Psychological Review, 78, 3-43.

(Manuscript received March 6, 1990; revision accepted for publication June 4,1990 .) 